\title{
Low Power Robust Computing
}

\author{
Trevor Mudge \\ University of Michigan, Ann Arbor \\ tnm@eecs.umich.edu
}

\begin{abstract}
In a recent speech Intel founder Andrew Grove argued that Moore's law will not slow down for at least a decade. By that time, integrated circuits will have feature sizes of 30 nanometers, allowing for integration of billions of devices on a single die and enabling unforeseen computational capabilities. However, with growing levels of integration, power densities will also skyrocket to hundreds of Watts. In fact, Grove cites power consumption as a major show stopper with off-state current leakage "a limiter of integration."

In addition to the power consumption crisis, aggressively scaled feature sizes also result in increased process variability and poor reliability. Hence, Grove mentions that at 30nm design will enter an era of "probablisitic computing," with the behavior of logic gates no longer being deterministic. To take advantage of scaling, it will be necessary to compute in the presence of various types of errors.

Our talk will present recent results in robust low power computing. The perspective will be microarchitectural: what can the microarchitect do to reduce the dependency on power and improve robustness. We will discuss recent academic and commercial proposals to limit power consumption. Finally, we will review some techniques to improve robustness based on recent ideas in timing speculation exemplified by our Razor research.
\end{abstract}

Biography: Trevor Mudge is the Bredt Family Professor of Electrical Engineering and Computer Science at the University of Michigan. He received a Ph.D. in computer science from the University of Illinois, Urbana-Champaign. His research interests include computer architecture, CAD, and compilers. He has chaired over 30 theses and authored over 250 articles in these research areas. In addition, he is the founder of Idiot Savants, a chip-design consultancy. He is a Fellow of the IEEE and a member of the ACM, the IEE, and the British Computer Society. 